\title{
Probing luminescence centers in Na rich feldspar
}

\section{Prasad, Amit Kumar; Lapp, Torben; Kook, Myung Ho; Jain, Mayank}

\section{Published in:}

Radiation Measurements

Link to article, DOI:

10.1016/j.radmeas.2016.02.033

Publication date:

2016

Document Version

Peer reviewed version

Link back to DTU Orbit

Citation (APA):

Prasad, A. K., Lapp, T., Kook, M. H., \& Jain, M. (2016). Probing luminescence centers in Na rich feldspar. Radiation Measurements, 90, 292-297. https://doi.org/10.1016/j.radmeas.2016.02.033

\section{General rights}

Copyright and moral rights for the publications made accessible in the public portal are retained by the authors and/or other copyright owners and it is a condition of accessing publications that users recognise and abide by the legal requirements associated with these rights.

- Users may download and print one copy of any publication from the public portal for the purpose of private study or research.

- You may not further distribute the material or use it for any profit-making activity or commercial gain

- You may freely distribute the URL identifying the publication in the public portal

If you believe that this document breaches copyright please contact us providing details, and we will remove access to the work immediately and investigate your claim. 


\title{
Probing luminescence centers in Na rich feldspar
}

\author{
A. K. Prasad", T. Lapp, M. Kook, M. Jain \\ Center for Nuclear Technologies, Technical University of Denmark, DTU Ris $\varnothing$ Campus, Roskilde 4000, \\ Denmark \\ (*Corresponding author: $\underline{\text { akpr@dtu.dk) }}$
}

\begin{abstract}
:
In contrast to the detailed investigations on the dosimetric electron trap in feldspar only little has been done to understand the luminescence centers. We use a comparison of multiple spectroscopic techniques, site selective photoluminescence spectroscopy and time resolved measurements to further our understanding of the luminescence mechanisms and recombination sites, in a sample of $\mathrm{Na}$ rich plagioclase feldspar (oligoclase). Both the UV and violet-blue emissions show resonant excitations arising from a distribution of energy levels. We propose, contrary to the general understanding, that the green emission may not arise from $\mathrm{Mn}^{2+}$ in our sample and that photoionisation of this centre may be possible by excitation to the band tail states. The deep red emission is tied to the $\mathrm{Fe}^{3+}$, and the exponential rise in the $\mathrm{UV}$ excitation efficiency of this centre is discussed in the context of the band-tail model.
\end{abstract}

\section{Introduction}

The photoemissions from feldspars are widely used in retrospective dosimetry and optical dating of geological and archaeological events (Huntley et al., 1985; Jain et al., 2014; Krbetschek et al., 1997). Feldspars are ubiquitous in nature, and the dosimetric signal measured as infra-red stimulated luminescence (IRSL) shows growth up to $1 \mathrm{kGy}$ dose range, allowing reconstruction of geological history up to about 0.5 Ma. However, this signal suffers from anomalous, athermal fading. A large body of work exists on the dosimetric, electron trapping centre which gives rise to this IRSL signal (Hutt et al., 1988; Poolton et al., 1989; Jain and Ankjærgaard, 2011; Anderson et al., 2012; Kars et al., 2013, etc.); these studies include both the tunnelling behaviour and the mapping of the energy level within the band gap. In contrast, only little has been done to characterise the recombination centers. Based on spectral characteristics of feldspars (Krbetschek et al., 1997), it is inferred that the UV emission arises from an intrinsic defect (Guinea et. al, 1996), 
the blue emission, common to most feldspars, from a hole-bearing oxygen atom adjacent to two Al atoms (Al-O-Al) (Finch and Klein et al., 1999), the green-orange emission, characteristic of some Na-feldspars, from $\mathrm{Mn}^{2+}$ luminescence centre occupying Ca sites (Geake et al., 1977), and the deep red emission band from tetrahedral $\mathrm{Fe}^{3+}\left({ }^{4} \mathrm{~T}_{1} \rightarrow{ }^{6} \mathrm{~A}_{1}\right.$ transition) substituting for $\mathrm{Al}^{3+}$ ( Geake et al., 1977; Telfer et al., 1975; White et al., 1986). A more comprehensive characterisation of the recombination centers is lacking. For example, there is little understanding of the distribution as well as the positions of the energy levels of these defects within the band gap. Similarly, there is little information on the characteristic fluorescence/phosphorescence lifetimes of these emissions.

The objective of this study is to further understand the luminescence transitions within the recombination centers in feldspars, and explore their energy structure and distribution within the bandgap. A special focus is given on the green and deep red emission. We use a combination of probing methods: X-rays in $\mathrm{keV}$ regime (X-ray excited optical luminescence: $\mathrm{XEOL}$ ), $\mathrm{Sr}^{90} / \mathrm{Y}^{90}$ beta particles (radioluminescence: RL) and UV-VIS-IR photons (photoluminescence: PL). These beta and X-ray excitations directly ionize the host lattice because the incident energy is much higher than the band gap of the feldspars $\sim 7.7 \mathrm{eV}$ (Poolton et al., 2006) leading to electron-hole recombination at the luminescence centers. In contrast, the optical excitation (PL; 1.9eV - 4.86eV) allows the possibility of probing the energy structure of these centers and possibly their disposition within the band gap using site selective spectroscopy. We also report on the luminescence lifetimes on microsecond and millisecond time scales characteristic of these centres.

\section{Instrumentation and sample}

We measured a set of feldspar museum specimens reported previously by Ankjærgaard et al. (2009) and Morthekai et al. (2013) using RL and PL. Of these a representative oligoclase sample (Oligoclase 1 of Morthekai et al., 2013) was chosen for detailed studies presented here as this sample had relatively higher optical sensitivity compared to the other samples, and showed all the typical bands reported for feldspars (Krbetschek et al., 1997). The main cationic constituents of the sample are (in molar \%): Na-73.7, Ca-18.9 and K-7.4; their concentrations were measured using inductively coupled plasma mass spectrometry (ICP-MS; Morthekai et al., 2013). The grain size used in the present studies was in the range of $90-180 \mu \mathrm{m}$. 
To measure the RL, the sample was mounted with a silicon oil into the steel cup and placed in the Ris $\varnothing$ TL/OSL Reader (Model DA 20) equipped with a $\mathrm{Sr}^{90} / \mathrm{Y}^{90}$ beta source (dose rate $0.1 \mathrm{~Gy} / \mathrm{s}$ ). The signal was collected through a fiber bundle; the circular $(3 \mathrm{~mm} \phi)$ end of the bundle looked at the sample under the beta source, while the other end arranged in an array of two rows was coupled to the Shamrock 193i spectrograph which dispersed the spectra on a high sensitivity EMCCD (iXon Ultra 888). The RL spectra were recorded from $250 \mathrm{~nm}$ to $1050 \mathrm{~nm}$ by setting the $300 \mathrm{lines} / \mathrm{mm}$ grating in four overlapping positions. The RL spectra were corrected for the spectral functions of grating, optical fibre and EMCCD.

The photoluminescence spectra were measured using Horiba Fluorolog-3 spectrofluorometer. A 450W Xenon CW lamp was used for excitation within a wavelength range of approximately 255 $\mathrm{nm}-600 \mathrm{~nm}$, with required wavelengths selected using a double grating Cherny-Turner excitation monochromator. Emission spectra in the range of $290 \mathrm{~nm}-600 \mathrm{~nm}$ were measured with a double grating Cherny-Turner spectrometer equipped with a photomultiplier tube (PMT). Spectra from 620 $\mathrm{nm}$ to $1000 \mathrm{~nm}$ were measured with Jobin-Yvon, HR-320 spectrometer (300 lines/ mm grating) coupled to a liquid nitrogen cooled CCD detector, because of better efficiency of the CCD compared to PMT in the red-IR region. All spectra were corrected for the instrument response and photon flux. Appropriate long-pass filters were placed between the sample and the emission monochromator to remove second order diffraction of the primary excitation light. The PL phosphorescence decay was measured with the pulsed Xe-lamp placed in the same housing as the CW-lamp. The required wavelength of the excitation pulses was selected with the excitation monochromator and emission was recorded with the emission monochromator. The integration time of the emission collection was $100 \mathrm{~ms}$ and the initial delay of the system was $0.05 \mathrm{~ms}$; all the measurements used flash counts of 100 to improve counting statistics.

XEOL was measured using a $40 \mathrm{kV}$ X-ray tube installed on Horiba Fluorolog-3 spectrofluorometer. The X-ray tube was operated at $39.7 \mathrm{kV}$ anode voltage and $95.7 \mathrm{~mA}$ anode current, and sample chamber pressure of $2.3 \times 10^{-4} \mathrm{mB}$ (vacuum was used because of interlock constraints). All low temperature luminescence measurements were accomplished in the same system using variable temperature (7-300K) closed loop He cryostat operated with a sample chamber pressure at 2.4 x $10^{-}$ ${ }^{5} \mathrm{mB}$, integrated into the Horiba emission/excitation spectrofluorometer. The proportional integralderivative (PID) temperature control is provided through a Lakeshore (model 335) control unit. The 
sample grains were directly mounted on a copper-based sample holder (a cold finger) with the help of carbon tape.

\section{Comparison of the XEOL, PL and RL spectra}

Figure 1 shows comparison of normalised XEOL, PL and RL spectra of our oligoclase sample. Four broad emission peaks can be observed at around 1.70, 2.2, 3.1 and $3.8 \mathrm{eV}$. The XEOL and the RL data are broadly similar, suggesting the same mechanism for both these cases; this is not surprising since both the $40 \mathrm{kV}$ X-rays and the high-energy beta particles (mean energy $600 \mathrm{keV}$ ) have energy higher than the band gap and access the entire sample volume. However, there is a hump at about $2.5 \mathrm{eV}$ in the RL spectra which is not present in XEOL; it needs to be confirmed if this hump is real or it is a measurement artefact.

In contrast to the RL/PL spectra, the relative intensity of the $2.2 \mathrm{eV}$ peak is strongly subdued in the PL spectra, suggesting that the optical cross-section of the green emission centre is small compared to the other centers; this, however, is not an issue in RL and XEOL as the electron relaxation occurs from the conduction band in the latter mechanisms. The spectral bands observed in our work are in good agreement with the previous studies (Krbetschek et al., 1997).

\section{Site selective photoluminescence spectra and luminescence lifetimes}

PL excitation and emission spectra were measured to get further information about the energy structure of the defects giving rise to the above mentioned emission bands. The results are presented in Figure 2(a)...(d) and discussed below:

UV emission: The black curve in Figure 2(a) shows excitation spectrum for the emission at $3.86 \mathrm{eV}$. There is a broad, well defined excitation peak at $4.27 \mathrm{eV}$ with a FWHM of $\sim 0.4 \mathrm{eV}$. The blue curve shows the corresponding emission spectrum excited at $4.27 \mathrm{eV}$ consisting of two overlapping peaks at $3.86 \mathrm{eV}$ and $3.67 \mathrm{eV}$. Figure $2 \mathrm{e}$ shows the phosphorescence decay of the emission at $3.86 \mathrm{eV}$ excited at $4.28 \mathrm{eV}$. We attempted fitting these data with a linear combination of discrete exponentials, a stretched exponential, or a Becquerel function; of these only the discrete exponentials function gave satisfactory fit to all the phosphorescence data presented in this study. The UV phosphorescence decay curve was best fitted with three exponentials with characteristic lifetimes $0.0023 \mathrm{~ms}, 0.046 \mathrm{~ms}$ and $0.36 \mathrm{~ms}$. The shortest lifetime may not be resolved accurately as the maximum data resolution in our system is 1 microsecond. 
Violet-blue emission: The excitation spectrum (black curve) for the $3.04 \mathrm{eV}$ emission is plotted in Figure2 (b); the spectrum appears as a broad asymmetric peak with a FWHM of $\sim 1 \mathrm{eV}$. It is likely that this peak consists of several overlapping peaks. The emission spectrum (the blue curve) was obtained using excitation at $3.69 \mathrm{eV}$; it is broad and asymmetric, and peaks at $3.06 \mathrm{eV}$. The phosphorescence decay (excitation at $3.69 \mathrm{eV}$ and emission at $3.04 \mathrm{eV}$ ), slightly slower compared to the UV emission, was best fitted to a sum of 3 exponentials (Figure 2f) with decay times $0.0043 \mathrm{~ms}$, $0.095 \mathrm{~ms}$ and $0.21 \mathrm{~ms}$.

Green emission: Figure 2c shows the excitation spectrum (black curve) for the emission at $2.21 \mathrm{eV}$. The excitation spectrum is largely flat with minor peak structures. There is a sharp decrease in excitation efficiency below $2.7 \mathrm{eV}$ and an increase above $4.2 \mathrm{eV}$; no discrete resonance peaks are observed as in contrast to the other emissions. Interestingly, the emission peak (blue, green and red curves in Figure 2c), shows a shift to the red side with a decrease in the excitation energy (discussed in detail in the next section). The phosphorescence decay curves were measured using the excitation energies corresponding to emission peaks, for e.g. excitation at $3.26 \mathrm{eV}$ for emission at $2.75 \mathrm{eV}$, and excitation at $2.95 \mathrm{eV}$ for emission at $2.44 \mathrm{eV}$ and so on (Figure $2 \mathrm{~g}$ ). It was observed that the different emissions have similar characteristic decay. The fitting analysis for the excitation-emission combination $2.95 \mathrm{eV}$ and $2.44 \mathrm{eV}$, respectively gave lifetimes of $0.0087 \mathrm{~ms}, 0.10 \mathrm{~ms}$ and $0.66 \mathrm{~ms}$.

Deep red emission: The excitation spectrum (black curve) for the emission at $1.65 \mathrm{eV}$ is plotted in Figure 2(d) (with the logarithmic scale for the excitation intensity). There is an exponential rise in the excitation curve between $3.85-4.86 \mathrm{eV}$, and resonance peaks are observed at 3.28, 2.95 and $2.75 \mathrm{eV}$. The emission spectrum (blue curve) was measured with the $4.27 \mathrm{eV}$ excitation using the CCD channel, and shows a symmetric peak cantered at $1.7 \mathrm{eV}$. The phosphorescence decay for this emission was also measured with $4.27 \mathrm{eV}$ excitation (Figure $2 \mathrm{~h}$ ) and contained three exponential components with the lifetimes of $0.14,0.67$ and $2.3 \mathrm{~ms}$. In order to investigate the relationship between the decay kinetics and the emission, we compared the shapes of the fluorescence emission spectrum and the phosphorescence emission spectra obtained after various delays with respect to the excitation pulse. It is observed that the fluorescence and phosphorescence spectra are broadly similar; however, a closer examination shows that there a slight but systematic blue shift in the phosphorescence emission with greater delay (Figure 3). 


\section{Dependence of emission peak on the excitation energy in the green emission}

Based on the result in Figure 2c, we explored in detail the dependence of the emission spectra on the excitation energy. We observed that there is a systematic shift in emission maxima from 2.13 to $2.49 \mathrm{eV}$ with a concomitant increase in intensity, as the excitation energy is tuned from 2.38 to 2.88 $\mathrm{eV}$ (Figure 4). Within the range of these data there is an almost linear correlation between the emission and the excitation peak energy (Figure $4 \mathrm{a}$ inset). The normalized (by emission maxima) spectra in Figure $4 \mathrm{~b}$ show that the peaks become systematically narrower (FWHM) as they shift to a lower energy.

We further explored the behaviour of the green emission with changes in sample temperature. The same measurements as those presented in figure 4 were repeated at $7 \mathrm{~K}, 100 \mathrm{~K}$ and $295 \mathrm{~K}$ using the cryogenic attachment. Normalised spectra for each temperature for three different excitation energies (at 2.81, 2.63 and $2.43 \mathrm{eV}$ denoted by arrows with the same colour) are shown in figure 5a. The shapes of the $295 \mathrm{~K}$ (solid curve), $100 \mathrm{~K}$ (plus) and $7 \mathrm{~K}$ (circle) are broadly similar with minor deviations at the low energy end. This deviation is particularly conspicuous for the $2.43 \mathrm{eV}$ excitation, where a small hump at $\sim 1.93 \mathrm{eV}$ is observed in the $295 \mathrm{~K}$ and $100 \mathrm{~K}$ data.

A strong thermal quenching was observed in the green emission; this effect can be observed in the integrated counts (area under the emission peak) obtained after each excitation energy at different temperatures (Figure 5a, inset). There is a reduction in emission intensity by a factor of two due to increase in the sample temperature from 100 to $295 \mathrm{~K}$, and a relatively minor decrease from $7 \mathrm{~K}$ to 100K. Similarly, as observed in figure $4 \mathrm{a}$, there is an increase in emission intensity, and emission energy with excitation energy at each temperature. It is observed that the emission intensity increases exponentially with excitation energy, with a slope of $3.08 \mathrm{eV}^{-1}$ at $7 \mathrm{~K}$ and $100 \mathrm{~K}$, and 3.04 $\mathrm{eV}^{-1}$ at $295 \mathrm{~K}$ (figure $5 \mathrm{a}$, inset). We also compared the phosphorescence decay at $295 \mathrm{~K}$ and $7 \mathrm{~K}$. There is a significant increase in the overall decay rate at room temperature compared to $7 \mathrm{~K}$, as demonstrated for the 2.69 and $2.81 \mathrm{eV}$ excitations (Figure 5b).

\section{Summary and Discussion}

RL and XEOL for the excitation energies described in this study, arise from electron-hole recombination through the delocalised bands (similar to OSL and TL), whereas PL occurs thorough 
selective excitation of a centre. A general similarity between the RL, XEOL, and PL spectra demonstrates that the same recombination sites must participate in both the delocalised (RL, XEOL) and localised mechanisms (PL); hence the results presented here are also relevant for dosimetric signals produced through delocalised transitions such as TL and OSL emissions. Based on the PL emission/excitation characteristics we conclude that both the UV and violet-blue emissions arise from deep localised defects within the bandgap, our present data however does not allow determination of the exact energy level. Nonetheless, the broad asymmetric bands suggest that there is distribution of energy levels either in the excited or the ground state or both. The FWHM of excitation spectra for the UV and violet-blue emission was $\sim 0.4 \mathrm{eV}$ and $1 \mathrm{eV}$, respectively, indicating the width of the corresponding energy level distributions. The phosphorescence decay ranges from few $\mu \mathrm{s}$ to hundreds of $\mu \mathrm{s}$, with an overall faster decay for the UV compared to the violet-blue emission. In comparison to the UV emission, the much wider excitation and emission peaks and the slower decay kinetics of the violet-blue emission are likely related to the distribution of these defects in the lattice. For example, it is possible that violet-blue emission may involve defect clusters, which lead to a flatter wider emission peak and slower decay because of charge transfer across defects.

The green emission is much stronger in XEOL and RL compared to PL suggesting that direct photo-excitation within our data range is relatively inefficient. The green-orange/red emission has typically been ascribed to $\mathrm{Mn}^{2+}$ in the literature (e.g., Krbetschek et al., 1997) due to the spin and parity forbidden ${ }^{4} \mathrm{~T}_{1} \rightarrow{ }^{6} \mathrm{~A}_{1}$ transition. This emission in the host lattice typically occurs with a lifetime of the order of tens of milliseconds within the $3 \mathrm{~d}$ shell, where the electrons are strongly coupled to lattice vibration and affected by crystal field strength and site symmetry (e.g. Shi et al., 2010). In our phosphorescence data, we observe the dominant lifetimes of the order of hundreds of microseconds to less than a fraction of a millisecond, which is not consistent with the interpretation that the green emission is arising from discrete $\mathrm{Mn}^{2+}$ defects. We, therefore, interpret that another unknown defect, rather than $\mathrm{Mn}^{2+}$ is responsible for the green emission in our sample.

The relatively flat excitation spectrum for the green emission indicates a distribution of excitation energies (continuous overlapping emissions); such a distribution could arise from the regions of clustered impurity, or because of the role of the band tail states. The interpretation of a distribution of states is supported by a strong dependence of emission energy and intensity on the excitation energy. A higher excitation gives rise to wider range of emission energies in comparison to the 
excitation to the lower energy band tail states; more specifically, the high energy emissions are systematically eliminated as we decrease the excitation energy (figure 4a). Measurements at cryogenic temperatures neither give higher resolution peaks, nor show a shift in the emission peak which is characteristic of the increasing crystal field effect on $\mathrm{Mn}^{2+}$. Instead the shape of the emission spectra at $7 \mathrm{~K}$ and $100 \mathrm{~K}$ is broadly similar to that at the room temperature, supporting that these emissions arise from a continuous distribution of states. The main effect of cooling is observed to be an increase in emission intensity with a simultaneous increase in the phosphorescence lifetime; this suggests that at higher temperatures relaxation may be occurring via lattice phonons, supporting a Mott-Seitz type (e.g., Pagonis et al., 2010) thermal quenching mechanism.

The excitation efficiency data may give insights into the energy of the defect centre responsible for the green emission. The excitation spectrum for the green emission shows a rise at $4.2 \mathrm{eV}$; however, this rise is not reliable because of the change in emission peak positions as a function of excitation energy. In fact there is a rapid increase of violet-blue and deep red emission for excitation energies $>3 \mathrm{eV}$, which makes it impossible to distinguish the characteristic green emission above this energy; thus we cannot conclusively map this defect with respect to the conduction band edge. However, the exponential rise in the emission intensity with excitation energy, at the rate of 3.08 $\mathrm{eV}^{-1}$ is insightful (figure 5a inset); the inverse is almost identical to the Urbach energy of $0.32 \mathrm{eV}$ estimated from a sodium feldspar sample by Poolton et al. (2009). Because of this remarkable similarity with our result, we interpret that the increase in excitation efficiency at higher energies results from the exponential increase in the density of the band tail states with an Urbach width of $0.32 \mathrm{eV}(=1 / 3.08)$ in our sample, rather than due to possible 'local clustering' of the responsible defect. The band tail states also explain the widening of the emission spectra with excitation energy.

The excitation spectra for deep red emission $(1.65 \mathrm{eV})$ have many resonant features which can be tied to the known transitions in $\mathrm{Fe}^{3+}$ (Poolton et al., 1996). The prominent phosphorescence decay is observed to be on the millisecond time scales confirming the spin forbidden nature ${ }^{4} \mathrm{~T}_{1} \rightarrow{ }^{6} \mathrm{~A}_{1}$ transition. Furthermore, the similarity of the fluorescence and phosphorescence spectra confirm that the emission is due to the forbidden transition of $\mathrm{Fe}^{3+}$; a slight systematic shift in the peak energy of the phosphorescence spectra with delay time with respect to the excitation pulse, suggests slightly different electronic environment of $\mathrm{Fe}^{3+}$ leading to a slightly different lifetimes as well as emission energies. This interpretation explains the fact that multiple exponentials are required approximate 
the phosphorescence decay of the red emission. We also observed that the excitation spectra have a continuum in the form of an exponential rise from 3.6 to $4.86 \mathrm{eV}$. This exponential rise is either related to the fine splitting and overlapping distribution of the higher excitation states of $\mathrm{Fe}^{3+}$ due to clustered impurities or, as in the case of green emission, it could be due to the access to the band tail states with an exponential distribution of the density of states. The characteristic energy for the exponential rise is estimated to be $\sim 0.23 \mathrm{eV}$ (inverse of the exponential constant of $4.3 \mathrm{eV}^{-1}$ obtained for data from 3.8 to $4.2 \mathrm{eV})$, which is similar to that derived for the green emission $(=0.32$ $\mathrm{eV})$. The difference in the exponential constants for the two emissions could be reconciled with the fact that the distribution of band-tail states may differ slightly in the vicinity of different impurities occupying different positions in the lattice. Clustered impurities, for example, possible due to high concentration of $\mathrm{Fe}^{3+}$, may significantly affect the band tail states by altering the local lattice structure. Equally, impurity clusters could lead to the formation of charge transfer bands within the forbidden gap (sub-bands), which could give rise to a similar exponential trend. Further research is required to differentiate between the two models; however, given the similarity of the exponential constants for the green and the red emission we currently favour the band tail model.

\section{Conclusion}

We help further constrain the luminescence model of feldspars based on multiple spectroscopic techniques and time resolved measurements. Our data suggest that the different emissions (UV, violet-blue, green, deep red) arise from the different sites, and that there occurs a distribution of energy levels for each emission. We conclude that the green emission is likely not related to $\mathrm{Mn}^{2+}$ in our samples, whereas the deep red emission is related to the $\mathrm{Fe}^{3+}$. We interpret that the exponential increase in the excitation efficiency of the green and red emission centres is due to direct transition to the band tail states.

\section{Acknowledgement}

We thank the two anonymous referees for their critical input. Dr. N. R. J. Poolton is thanked for discussions during the early phase of this work. 


\section{Figure Captions}

Figure 1: A comparison of the XEOL (black), PL (Red) and RL (blue) spectra. In the case of XEOL and PL, the solid curves are the data collected through the PMT channel, whereas the dotted curves are collected using the CCD channel. A $610 \mathrm{~nm}$ long pass filter was used to avoid excitation scattered light for the collection of CCD data.

Figure 2: Site selective excitation and emission spectra, and phosphorescence decay data of $\mathrm{Na}$ feldspar. a-d: the black curves are the excitation spectrum while the coloured curves are emission spectra. The excitation spectrum in (d) is plotted on the log intensity scale while all the others are on the linear scale with arbitrary units. The emission spectra are obtained with the excitation energy marked by the arrow. The excitation spectra are obtained by fixing the emission in the narrow window shown as the dotted vertical line. (e) to (h) phosphorescence decay curves corresponding to each emission plotted on the left figures. The excitation and emission energies used in timeresolved measurements are mentioned in the legends.

Figure 3: Comparison of fluorescence and phosphorescence spectra of the deep-red emission using $4.27 \mathrm{eV}$ excitation. The black curve shows fluorescence spectra measured with continuous wave (CW) Xe-lamp excitation. The other curves represent phosphorescence emission spectra measured after a delay of $0.5,1,2,4$ and $8 \mathrm{~ms}$ with respect to excitation pulse. There is a systematic shift of the peak emission with delay as can be clearly observed on the higher energy limb. We used excitation and emission slit widths of $10 \mathrm{~nm}$ for phosphorescence measurement, in comparison to $2 \mathrm{~nm}$ for fluorescence measurement, to obtain a high signal-to-noise ratio.

Figure 4: The dependence of the emission peak energy of the green band on the excitation energy. (a) Excitation dependent emission spectra. The excitations were done at approximately every 0.05 $\mathrm{eV}$ between $2.38 \mathrm{eV}$ (corresponding emission curve in black) and $2.88 \mathrm{eV}$ (corresponding emission curve in yellow). The inset shows the emission peak energy vs. the excitation energy. (b) Normalized curves of the data in (a). 
Figure 5: (a) The emission spectra at $7 \mathrm{~K}, 100 \mathrm{~K}$ and room temperature (RT) represented by open circle, plus and solid line, respectively. These emission spectra are obtained using excitation with $2.81 \mathrm{eV}$ (black), $2.63 \mathrm{eV}$ (blue) and $2.43 \mathrm{eV}$ (red) light, indicated by the same coloured downward facing arrows. The inset shows area under the emission peak for different excitations at $7 \mathrm{~K}, 100 \mathrm{~K}$ and $295 \mathrm{~K}$ sample temperatures; note that the y axis is on a logarithmic scale. (b) Phosphorescence decay data shown for the excitation, emission and temperature conditions as mentioned in the legend. 


\section{References:}

Andersen, M. T., Jain, M., Tidemand-Lichtenberg, P., (2012). Red-IR stimulated luminescence in K-feldspar: Single or multiple trap origin? Journal of Applied Physics, 112, 043507.

Ankjærgaard C., Jain M., Kalchgruber, R., Lapp, T., Klein, D., McKeever, S.W.S., Murray, A.S., Morthekai, P., (2009). Further investigations into pulsed optically stimulated luminescence from feldspars using blue and green light. Radiation Measurements 44, 576-581.

Finch, A. A., and Klein, J., (1999). The causes and petrological significance of cathodoluminescence emissions from alkali feldspars. Contrib. Mineral Petrol., 135: 234-243.

Garcia-Guinea, J., Townsend, P. D., Sanchez-Munoz, L., Rojo, J. M., (1999). Ultraviolet-blue ionic luminescence of alkali feldspars from bulk and interfaces. Journal of Physics and Chemistry of Minerals, 26, 658-66.

Geake, J. E., Walker, G., Telfer, D. J., Mills, A. A.,(1977). The Cause and Significance of Luminescence in Lunar Plagioclase. Philosophical Transactions of the Royal Society A: Mathematical, Physical and Engineering Sciences, 285, 403-405.

Huntley, D.J., Godfrey-Smith, D.I.,Thewalt, M. L. W., (1985). Optical dating of sediments. Nature, London, 313, 105-107.

Hutt, G., Jaek, I., Tchonka, J., (1988). Optical dating: K-feldspars optical response stimulation spectra. Quaternary Science Reviews, 7, 381-385.

Jain, M., and Ankjærgaard, C., (2011). Towards a non-fading signal in feldspar: Insight into charge transport and tunnelling from time-resolved optically stimulated luminescence. Radiation Measurements, 46, 292-309.

Jain, M., (2014). Feldspar, Infrared-Stimulated Luminescence. Encyclopedia of Scientific Dating Methods, pages: 1-8, 2015, 
Kars, R. H., Poolton, N., Jain, M., Ankjærgaard, C., Dorenbos, P., Wallinga, J., (2013). On the trap depth of the IR-sensitive trap in Na- and K-feldspar. Radiation Measurements, 59, 103-113

Krbetschek, M. R., Goetze, J., Dietrich, A., Trautmann, T., (1997). Spectral information from minerals relevant for luminescence dating. Radiation Measurements, 27, 695-748.

Morthekai, P., Jain, M., Gach, G., Elema, D. R., Prip, H., (2013). Dependence of (anomalous) fading of infra-red stimulated luminescence on trap occupancy in feldspars. J. Lumin. 143, 704-709.

Pagonis, V., Ankjærgaard, C., Murray, A.S., Jain, M., Chen, R., Lawless, J., Greilich, S. (2010) Modelling the thermal quenching mechanism in quartz based on time-resolved optically stimulated luminescence. Journal of Luminescence 130, 902-909.

Poolton, N. R. J., and Bailiff, I. K., (1989). The use of LEDs as an excitation source for photoluminescence dating of sediments. Ancient TL, 7, 18-20.

Poolton, N. R J., Bøtter-Jensen, L., Johnsen, O., (1996). On the relationship between luminescence excitation spectra and feldspar mineralogy. Radiation Measurements, 26, 93-101.

Poolton, N. R. J., Mauz, B., Lang, A., Jain, M., Malins, A. E. R., (2006). Optical excitation processes in the near band-edge region of and feldspar. Radiation Measurements, 41, 542-548.

Poolton, N. R. J., Kars, R. H., Wallinga, J., Bos, A.J.J (2009) Direct evidence for the participation of band-tails and excited-state tunnelling in the luminescence of irradiated feldspars. J. Phys.: Condens. Matter 21 (2009) 485505

Shi, L, Huang, Y., Seo, H-J (2010) Red Shift and Unusual Band Narrowing of $\mathrm{Mn}^{2+}$ in NaCaPO4 Phosphor. J. Phys. Chem. A 2010, 114, 6927-6934.

Telfer, D. J., and Walker, G. (1975). Optical detection of $\mathrm{Fe}^{3+}$ in lunar plagioclase. Nature, 258, 694 - 695. 
White, W. B., Matsumara, M., Linnehan, D. G., Furukawa, T., Chandrasekhar, B. K., (1986). Absorption and luminescence of $\mathrm{Fe}^{3+}$ in single-crystal orthoclase. Am. Mineral. 71 1415-19. 
Figure1

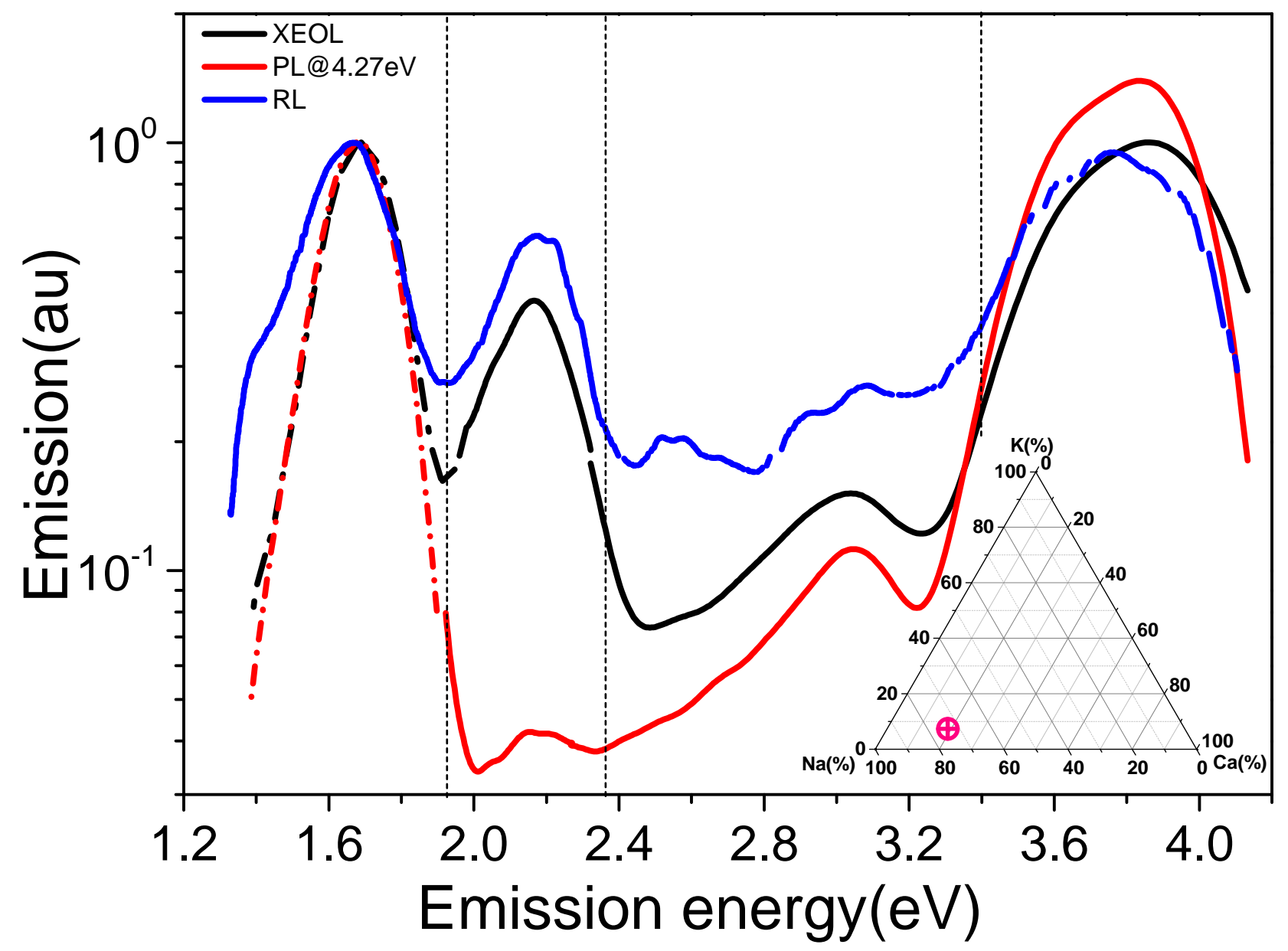


Figure 2
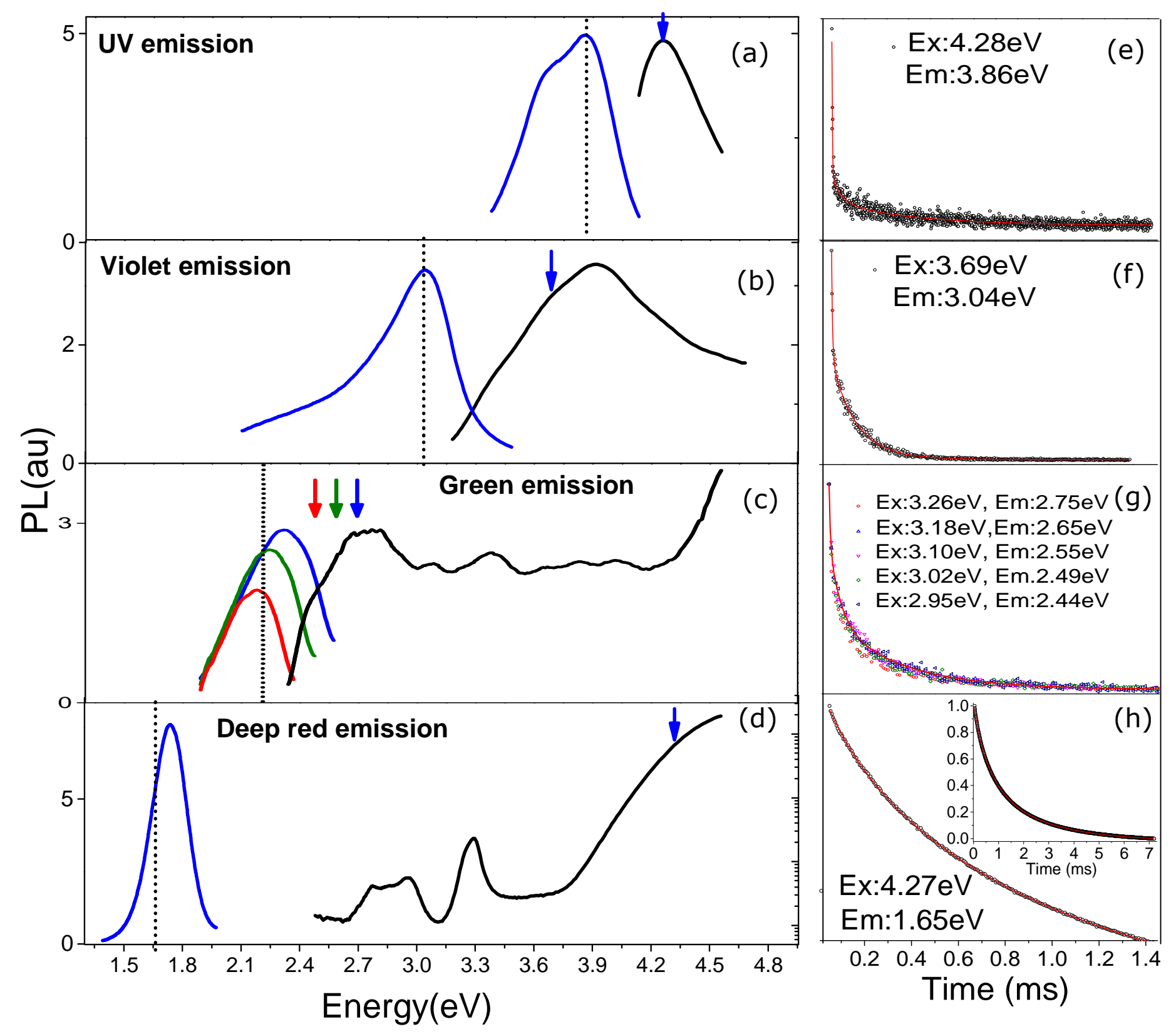

DTU Nutech, Technical University of Denmark 
Figure 3

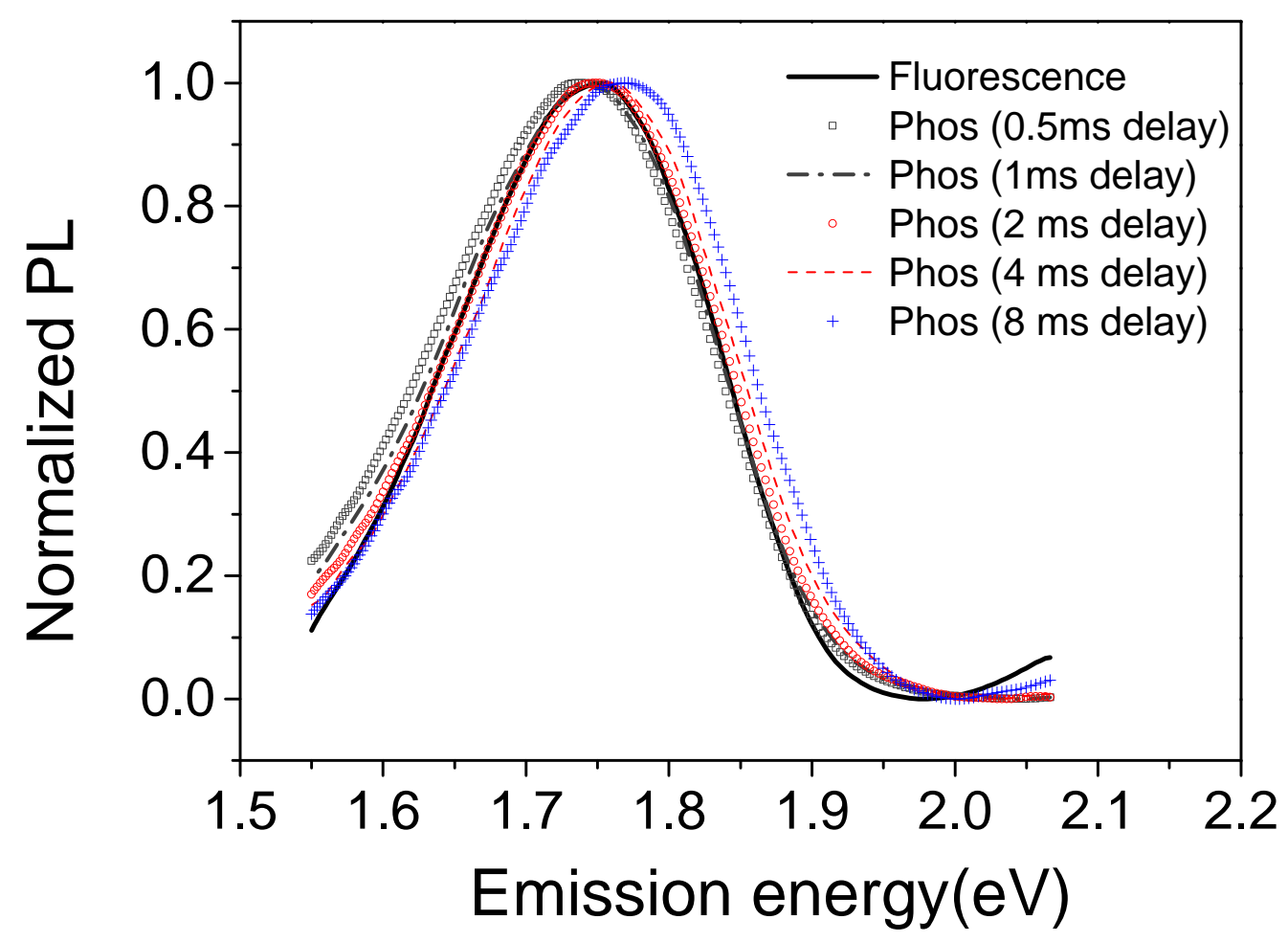


Figure 4

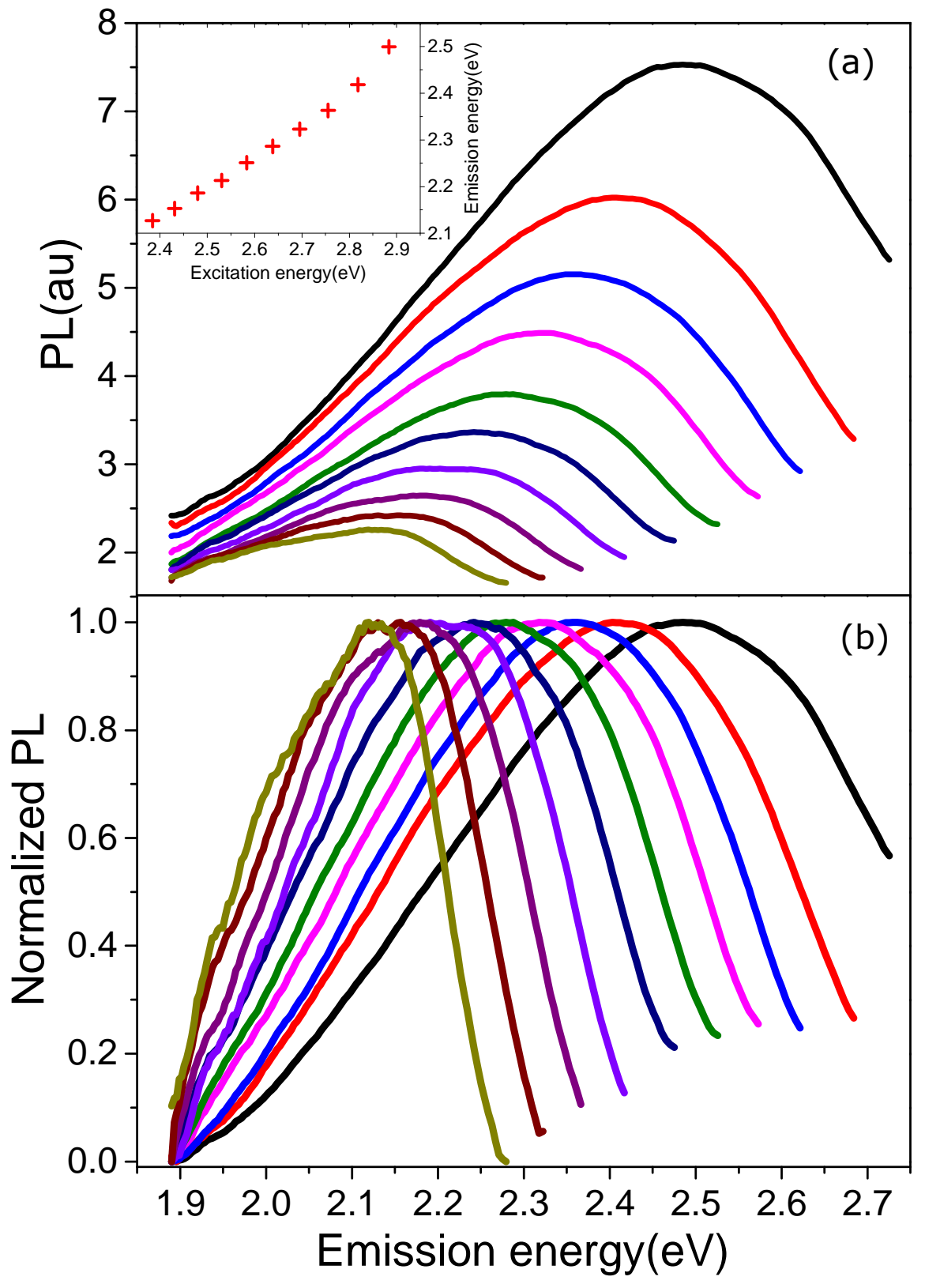


Figure 5
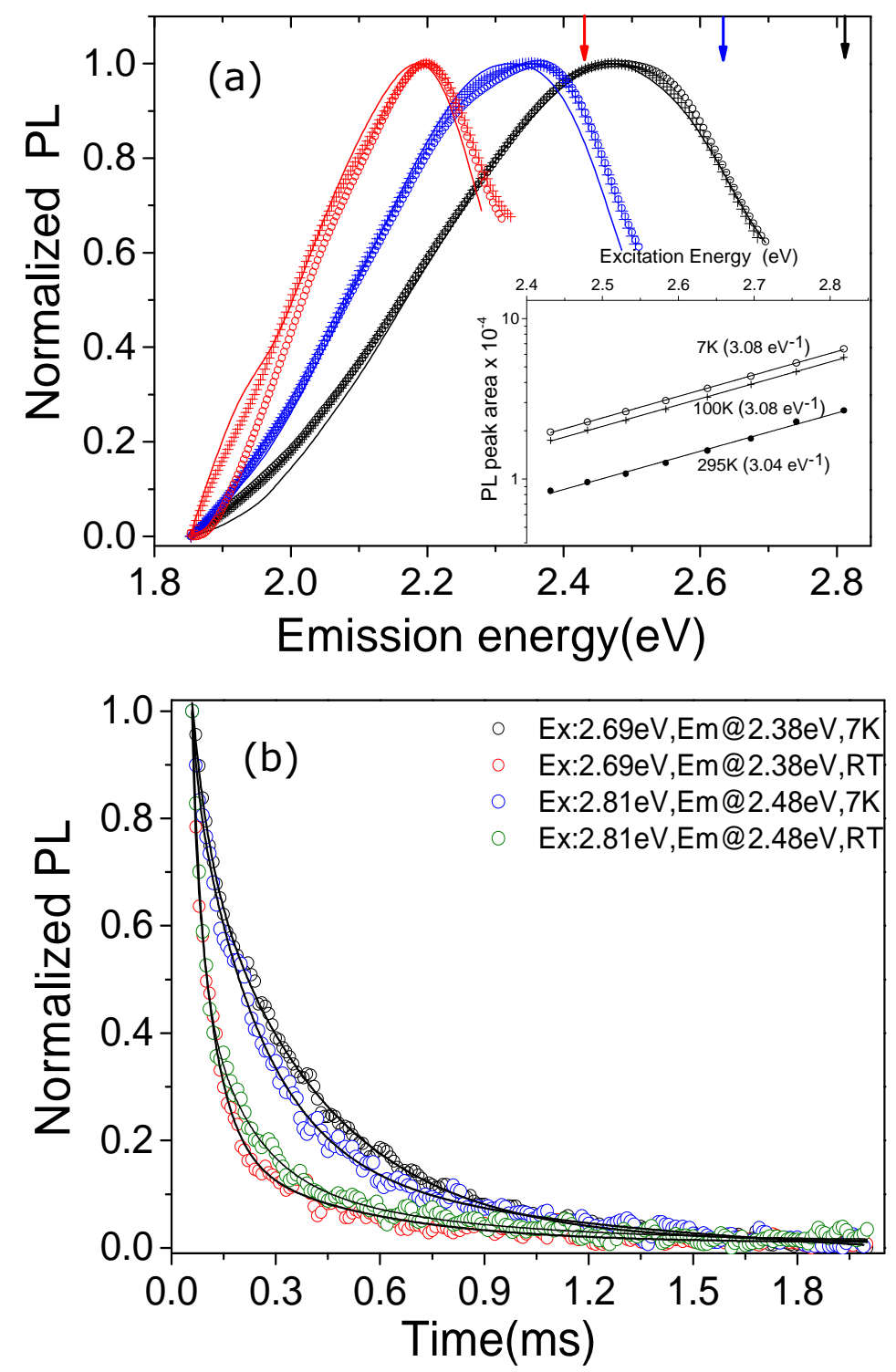
Highlights: Probing luminescence centers in Na rich feldspar

- The UV, violet-blue, green and deep red emissions characterised using site-selective spectroscopy.

- The green-yellow band shifts with the excitation energy and is not related to $\mathrm{Mn}^{2+}$.

- Band-tail states likely play a role in the green and deep red PL emissions. 